\title{
Auto-correlation analysis of wave heights in the Bay of Bengal
}

\author{
Abhijit Sarkar*, Jignesh Kshatriya** and K SAtheesan ${ }^{\dagger}$ \\ Meteorology $\&$ Oceanography Group, Space Applications Centre, ISRO, Ahmedabad 380 015, India. \\ *e-mail: sarkar_abhi2000@yahoo.com \\ **e-mail: k_jignesh@ipdpg.gov.in \\ $\dagger$ †-mail: $k_{-}$satheesan@rediffmail.com
}

Time series observations of significant wave heights in the Bay of Bengal were subjected to autocorrelation analysis to determine temporal variability scale. The analysis indicates an exponential fall of auto-correlation in the first few hours with a decorrelation time scale of about six hours. A similar figure was found earlier for ocean surface winds. The nature of variation of autocorrelation with time lags was also found to be similar for winds and wave heights.

\section{Introduction}

Temporal variability of environmental parameters reveals the scales of the processes. Its knowledge provides insight into prediction potentials of these parameters. The knowledge of time scales also improves the effectiveness of data assimilation in numerical models and validation exercises of physical parameters obtained by different means.

An auto-correlation analysis of ocean surface winds based on available time series over the Bay of Bengal was reported in our earlier work (Sarkar et al 2002). The present note attempts to carry out a similar exercise for ocean wave heights, a parameter that is primarily wind driven. Variability of these parameters is particularly important for the monsoon season and over the north Indian Ocean, the cradle of the monsoon systems. Because of the paucity of long time series data of oceanic winds and waves over the Indian Ocean, such an analysis has not been reported earlier.

\section{Data}

Several deep-sea and shallow water moored buoys have been functional in the north Indian Ocean since 1997 (Premkumar et al 2000) under the National Data Buoy Program (NDBP). No single buoy however has functioned continuously. In the present study, we have used wave and surface wind data measured by the deep-sea buoys operating in the Bay of Bengal at $18.0^{\circ} \mathrm{N}$ lat $/ 88.1^{\circ} \mathrm{E}$ long. The wind sensor was installed at $\sim 3 \mathrm{~m}$ above sea surface. The reported wind magnitudes are averages of 600 samples (measurements acquired over 10 minutes with sampling speed of 1 sample/second). The sensor used in the measurement of significant wave height is an inertial altitude heading reference system with dynamic linear motion measurement capability. Its accuracy is $\pm 20 \mathrm{~cm}$ and resolution $1 \mathrm{~cm}$. Normally the measurements are made every three hours. However, the data of wind speeds (WS) and significant wave height (SWH) used in our study are hourly observations, acquired as a part of a special campaign. The total duration of the time series for waves is 312 hours, while for winds it is 240 hours, both series start on 14 May 1999.

\section{Correlation analyses}

The hourly time-series of significant wave height consists of 312 observations shown in figure 1 . The

Keywords. Significant wave height; auto-correlation; temporal variability.

J. Earth Syst. Sci. 115, No. 2, April 2006, pp. 235-237

(C) Printed in India. 

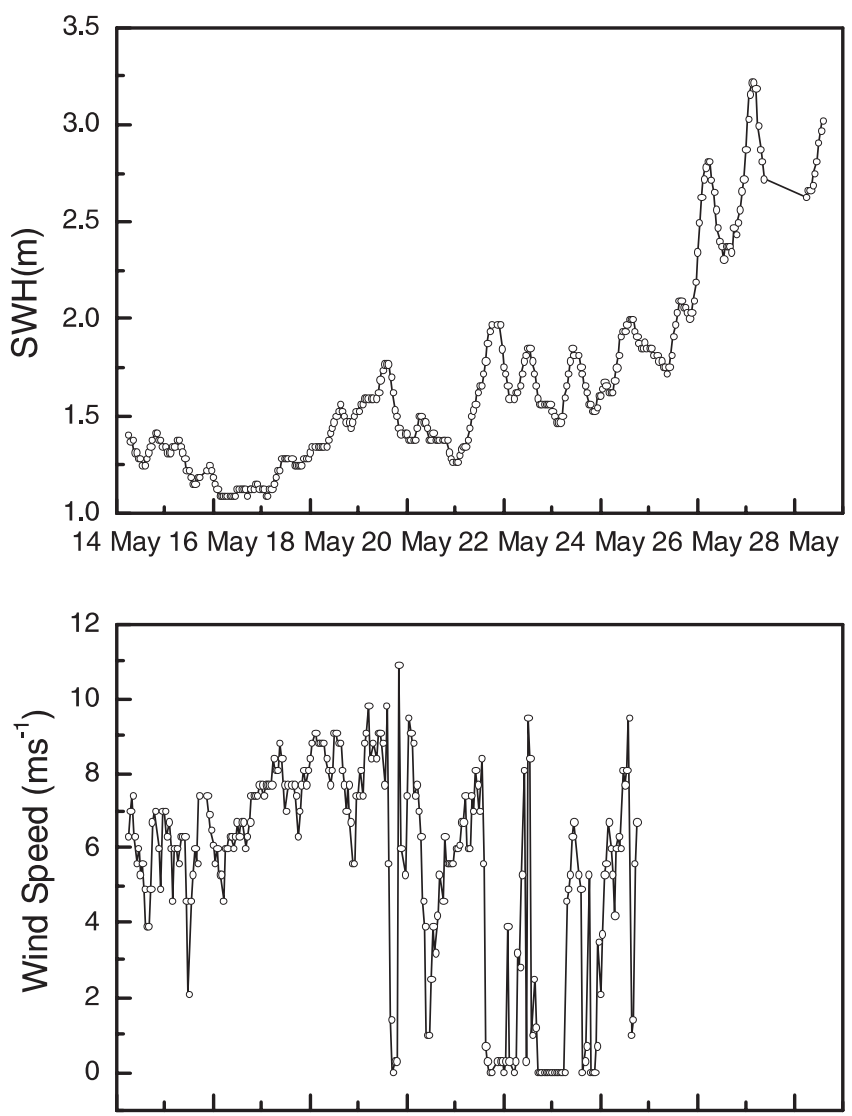

14 May 16 May 18 May 20 May 22 May 24 May 26 May 28 May

Days

Figure 1. Time series of SWH (in meters) and wind speed (in $\mathrm{m} / \mathrm{s}$ ).

analysis carried out earlier by us, with hourly time series observations of surface winds (Sarkar et al 2002) consisted of 240 observations (also starting on 14 May 1999 in the bay). The wind speed time series is also reproduced in figure 1 for the sake of completeness. The time-series of significant wave heights were first subjected to an auto-correlation analysis. Assuming the time series to be stationary, one can compute the auto-correlation function of the time series using the formula (Anderson 1994; IMSL 1991):

$$
\rho(k)=\frac{\sigma(k)}{\sigma(0)}
$$

where $k$ is the lag and $\sigma(k)$ is the auto-covariance function for lag $k$, and is given by

$$
\sigma(k)=\frac{1}{n} \sum_{t=1}^{n-k}\left(X_{t}-\mu\right)\left(X_{t+k}-\mu\right)
$$

where $\mu$ is the mean of the time series.

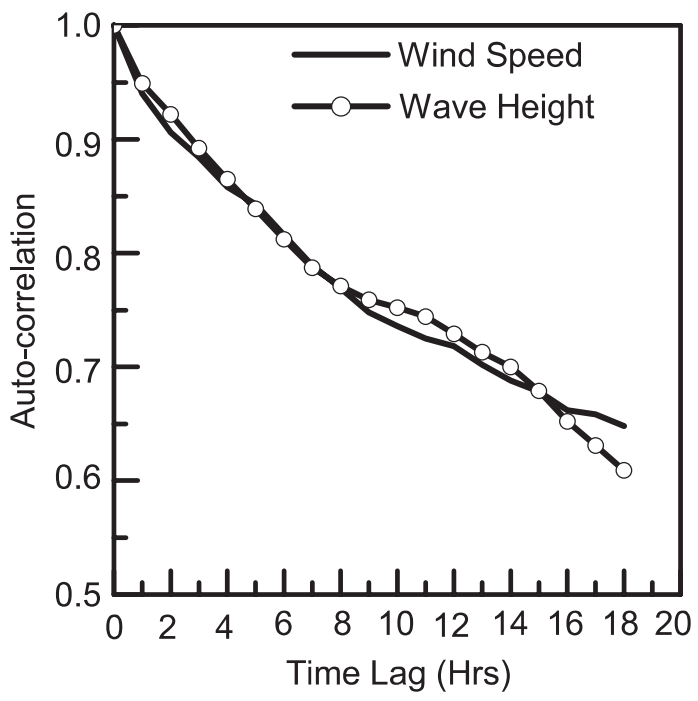

Figure 2. Variation of auto-correlation of significant wave height and wind speed with time lag (length of SWH series is 312 hours, and that of WS is 240 hours).

The auto-correlation values were computed for different spans of time starting with six hours. The computation showed an approach to convergence when the number of observations exceeds 200 .

\section{Results and discussions}

Figure 2 shows the variation of auto-correlation with time lag with all 312 hours' observations for wave height. Variation of auto-correlation for wind speed reported in Sarkar et al (2002) has also been shown in the figure for comparison. The nature of the fall of auto-correlation with time lag for both WS and SWH are identical. This is a characteristic, which is not apparent from the time series plot. The pattern of variation with time lag up to about eight hours is seen to be quite close to an exponential function and the subsequent analyses were restricted to time lags of up to eight hours. The e-folding value in the case of significant wave heights is about six hours, a value close to the e-folding value for surface wind speed (Sarkar et al 2002). The difference between the auto-correlation of SWH and that of WS is that, for SWH the auto-correlation falls to a value of $\sim 0.9$ in about 2.5 hours (as against 2 hours for WS). The wave field thus is slightly slower in losing its auto-correlation magnitude. The present study demonstrates that the temporal coherence scale of SWH is very similar to that of WS in our study area. Hence, in data assimilation/validation experiments with SWH, a weighting function with a threshold value between 0.5 and 3 hours may be designed. 


\section{References}

Anderson T W 1994 The statistical analysis of time series (New York: John Wiley \& Sons), 750 pp.

IMSL 1991 User's manual: Fortran subroutines for statistical analysis, pp. 684-688.
Premkumar K, Ravichandran M, Kalsi S R, Sengupta D and Gadgil S 2000 First results from a new observational system over the Indian Seas; Curr. Sci. 78 323-330.

Sarkar A, Basu S, Varma A K and Kshatriya J 2002 Autocorrelation analysis of ocean surface wind vectors, Proc. Indian Acad. Sci. 111 297-303.

MS received 4 June 2005; revised 14 October 2005; accepted 10 January 2006 